\title{
Why I blog and tweet
}

\author{
Skeptical Scalpel, MD
}

Received: 16 January 2015/ Accepted: 19 February 2015/Published online: 6 March 2015

(c) Canadian Anesthesiologists' Society 2015

As this free-standing editorial "goes to press", I will have been blogging and tweeting for more than five years. As I was nearing retirement from the practice of general surgery, I aspired to become a writer, and although I had authored more than 90 papers, review articles, and letters, I really didn't consider scientific writing as "writing". A willing mentor suggested that I start a blog and use Twitter to promote it. He also took the time to critique some of my early blog posts.

Here's what social media does for me. I learn from the people I follow and reciprocally from those who follow me. Using social media allows me to see what's going on in surgery and other specialties, to keep up with the news, connect with colleagues, and make new friends, and furthermore, to hone my writing skills. Blogging allows an outlet for me to vent my views, and I use Twitter for self-promotion. I receive valuable feedback from tweets and from comments on my blog site. The interactivity of social media is a key element that keeps me engaged.

The topics of my blog posts are mainly related to medical education, residency training, and medical research, but I do allow occasional forays into satire and social commentary. The blog has enabled me to talk about

This editorial is accompanied by a commentary. Please see Can J Anesth 2015; 62: this issue.

Cet éditorial est accompagné d'un commentaire. Veuillez consulter: Can J Anesth 2015; 62: ce numéro.

S. Scalpel, MD ( $\square)$

Any town, USA

e-mail: skepticalscalpel@hotmail.com

URL: http://skepticalscalpel.blogspot.ca/ things that I couldn't discuss when I was a residency program director for fear of retribution. My pseudonym has allowed me to be more candid. It's now at the point where the SkepticalScalpel brand is recognized by so many people that I am reluctant to give it up.

When I began my social media presence, my audience consisted of only a few friends, my adult children, and my wife - and I'm not even sure if she read all my blog posts. I continued posting new material three or four times a week, and slowly the readers came.

The impact of my blog has been surprising. For example, the tenth post I wrote, "Appendicitis: Diagnosis, CT Scans and Reality", has been viewed over 22,000 times - which I'm certain accounts for far more viewers than the total for all my medical publications combined. Indeed, isn't this the goal of all medical writers to have their material read by an expansive audience?

\section{Some tips for blogging}

For those considering starting their own medical blog, I have a few suggestions and comments. Edit carefully. I rarely post anything on the blog before revising it at least five times. This means doing something else for at least a few hours between revisions. When I revisit my draft, it looks less like my own writing and more like someone else's work that I'm more accustomed to editing - text that needs refining is easier to spot.

In addition to my personal blog, I have written for various medical websites, and one such site allows me to recycle some of my older posts. When I resubmit a previous post, I usually make a few changes - there is always room for improvement. Even so, I must temper that 
urge to keep revising - if I didn't, likely nothing would be posted to my blog.

I try to follow the novelist Elmore Leonard's rules for writing, ${ }^{1}$ especially this one: "If it sounds like writing, I rewrite it."

\section{What to write about}

At first, I had some trouble thinking of ideas for blog posts. Now my senses are keener. At least two or three times a day, I read something that could be a blog topic. Twitter is an excellent source of potential material. Links to possible subject matter can be stored in a simple document or in one of the several organizational applications such as Evernote (www.evernote.com).

Not every idea pans out. I currently have about 30 posts that I have started and not yet finished. I keep them in a file on my computer and occasionally revisit them hoping to resuscitate one or two. Sometimes it works.

When I first started my blog, I had someone else read my posts before they went online, which is a good way to find out if you are explaining things clearly. Sometimes what seems perfectly obvious and logical to a writer can be incomprehensible to a reader who does not have the same background or knowledge about the subject. Put another way, if a reader is unable to understand the meaning of the writer's post, it is primarily the fault of the writer - i.e., the lack of clarity in their writing.

If you intend to write about clinical topics and include vignettes about patients, you must be extremely careful about preserving the patients' anonymity; better still, get the patients' permission before you tell their stories. There are a number of anecdotes about unfortunate doctors who thought they had disguised a patient's identity adequately. Therefore, be sure to read and follow any rules that your institution may have regarding social media.

The brief attention span of most Internet users is well documented. I try to keep my posts to a maximum of 500 words. If you're planning to write more than that, it had better be riveting prose.

You can post to your blog as often as you like. Indeed, posting regularly gets readers in the habit of looking for your work. It's better to post good articles occasionally than mediocre ones frequently.

There are two main websites supporting blogging www.blogger.com (Blogger) and www.wordpress.org. Both websites are free and do not have limits on the amount of usable space. I started with Blogger and have stayed with it. Blogger is a bit confusing to use at first, but like most software, the more you use it, the more facile you become with it. Last year, at the Clinical Congress of the American College of Surgeons, I gave a lecture on how to establish a blog. Screenshots from that presentation are posted online. ${ }^{\mathrm{A}, 2}$

If you aren't sure whether you want to start your own blog, try submitting some guest posts to sites like KevinMD (http://www.kevinmd.com/blog/) or The Health Care Blog (http://thehealthcareblog.com/). Both sites welcome contributions.

\section{What about Twitter?}

Forget all you've heard about Twitter being a waste of time. If you tweet properly, it can be a major timesaver.

Twitter helps me to keep up with what is going on in the world, particularly in medicine. You can't read everything yourself. For example, there are more than 30 journals on the subject of anesthesiology alone. If you follow the right people, they can keep you up to date. You can use Twitter to control your own information feed.

Some medical specialties have found ways to maximize the value of Twitter. Emergency medicine physicians have created what they dub "FOAMed" (free open-access medical education). Tweets using the hashtag \#FOAMed share links to various papers, articles, and blogs. Several specialties, including general surgery, hold monthly Twitter journal clubs, with anyone being able to participate. A group of nephrologists just announced the founding of an internship in social media. ${ }^{2}$ The website digitalsociology.org lists many reasons why academics (also applicable to physicians) find Twitter useful, ${ }^{3}$ such as quick answers to questions, keeping up with things, networking, and so on.

The website GCFLearnFree.org has a simple tutorial showing how to set up a Twitter account. ${ }^{4}$ After you join Twitter, you can get a feel for it by "lurking" or simply by observing for a while, and you don't have to tweet unless you want to. Once you start tweeting, your posts are limited to 140 characters or less, which forces you to be concise and to learn to write effective headlines.

I use Twitter to drive traffic to my blog. For instance, for a day or two after I post something new on my blog, I post several tweets with a brief description of my posting and a link to the blog.

Sree Sreenivasan, former Chief Digital Officer for Columbia University, explained why the late movie critic Roger Ebert was a successful Twitter user. ${ }^{5}$ Ebert was interested in real life, engaged with others, and humble. He shared great content and attracted many followers.

\footnotetext{
A Scalpel S. Social Media: What's in It For Me and My Patients? October 28, 2014; Available from URL: https://s3.amazonaws.com/ z1g1.net/assets/acs2014/Skeptic-Scalpel-ACS-Talk.pdf (accessed January 2015).
} 
Sreenivasan also observed that social media has changed the way people view expertise. For example, he no longer relies solely on movie reviews by established critics. Instead, he uses Internet sites like Rotten Tomatoes (http:// www.rottentomatoes.com/) which compares reviews by critics with those of audience members.

Post-publication peer review is increasingly being discussed in science and medicine publication circles and is sure to expand. The medical search engine PubMed and journals such as the BMJ allow online comments about papers. A new site called PubPeer (http://pubpeer.com/) permits comments about any paper to be posted anonymously. Blog and Twitter postings easily lend themselves to this new form of post-publication peer review.

In short, blogging and tweeting can add value to your life, both medically and personally. Just be careful not to get distracted by cat videos.

\section{Pourquoi je blogue et utilise Twitter}

Quand cet éditorial va partir sous presse, j'aurai blogué et utilisé Twitter depuis plus de cinq ans. En approchant de ma retraite après une carrière de chirurgien généraliste, j'aspirais à devenir un écrivain et, bien que je soi déjà l'auteur de 90 articles, synthèses et lettres, je ne considérais pas vraiment la rédaction scientifique comme de « l'écriture ». Un mentor de bonne volonté m'a suggéré de commencer un blog et d'utiliser Twitter pour en faire la promotion. Il a également pris le temps de faire une critique de quelques-uns de mes premiers articles.

Et voici ce que font les médias sociaux pour moi. J'apprends des personnes que je suis et, également, de ceux qui me suivent. L'utilisation des médias sociaux me permet de voir ce qu'il se passe en chirurgie et dans d'autres spécialités, de rester au courant des nouvelles, d'être connecté à mes collègues, de me faire de nouveaux amis et, plus encore, d'affûter mes compétences d'écriture. Écrire un blogue me permet de faire connaître librement mes points de vue et j'utilise Twitter pour faire mon autopromotion. Je reçois des contributions intéressantes à travers Twitter et les commentaires postés sur le site de mon blogue. L'interactivité des médias sociaux est un élément clé qui me maintient engagé.

La thématique de mes articles tourne essentiellement autour de l'éducation médicale, la formation des résidents et la recherche médicale, mais je me laisse aller occasionnellement à la satire et aux commentaires sur la vie sociale. Le blogue m'a permis de parler de choses dont je n'aurais pas discuté lorsque j'étais directeur d'un programme de résidanat, par crainte de représailles. Mon pseudonyme m'a permis d'être plus « candide ». C'en est aujourd'hui au point que la marque SkepticalScalpel est reconnue par tellement de personnes que j'hésite à abandonner.

Quand j'ai commencé à être présent sur les médias sociaux, mon audience ne se composait que de quelques amis, de mes propres enfants adultes et de ma conjointe (et je ne suis même pas sûr qu'elle ait lu tous mes articles). J'ai continué à poster un nouveau contenu trois à quatre fois par semaine et les lecteurs sont lentement arrivés.

L'impact de mon blog a été surprenant. Par exemple, le dixième billet que j'ai écrit s'intitule «Appendicite: diagnostic, tomodensitométrie et réalité »: il a été vu plus de 22000 fois, ce qui représente de loin beaucoup plus de lecteurs que pour toutes mes publications médicales combinées. En fait, n'est-ce pas le but de tout rédacteur médical que de voir ses textes lus par un public de plus en plus important?

\section{Quelques conseils pour bien bloguer}

Pour ceux qui envisagent de commencer leur propre blogue médical, je ferais quelques suggestions et commentaires. Relisez attentivement. Je publie rarement quelque chose sur le blogue avant de l'avoir révisé au moins cinq fois. Cela signifie de faire quelque chose d'autre pendant au moins plusieurs heures entre chaque révision. Quand je reprends ma version préliminaire, cela ressemble moins à ma propre écriture et plus au travail de quelqu'un d'autre que j'ai plus l'habitude de réviser et, ainsi, le texte qui doit être amélioré est plus facile à détecter.

En plus de mon blogue personnel, j'ai écrit pour différents sites Web médicaux et l'un de ces sites m'autorise à recycler quelques-uns de mes plus anciens articles. Quand je soumets de nouveau un billet ancien, j'y fais habituellement quelques changements: il est toujours possible d'apporter des améliorations. Mais même dans ce cas, je dois freiner mon besoin de continuer à réviser; si je ne m'arrêtais pas, il est probable que je ne publierais rien.

J'essaie de suivre les règles d'écriture du romancier Elmore Leonard, ${ }^{1}$ en particulier celle-là: $\ll \mathrm{Si}$ cela ressemble à de l'écrit, je le réécris ».

\section{Écrire sur quoi?}

Au début, j'avais du mal à trouver des idées d'articles pour le blogue. Maintenant, mes sens sont plus affûtés. Il m'arrive de lire, au moins deux ou trois fois par jour, quelque chose qui pourrait être un sujet de billet. Twitter est une excellente source de matériels possibles. Des liens vers de possibles thèmes peuvent être conservés dans un 
simple document dans l'une des quelques applications organisationnelles comme Evernote (www.evernote.com).

Toutes les idées ne mûrissent pas. J'ai actuellement commencé une trentaine de billets qui ne sont pas encore finis. Je les conserve dans un dossier de mon ordinateur et je retourne les voir de temps à autre, dans l'espoir d'en ramener un ou deux à la vie. Parfois, cela marche.

Quand j'ai commencé mon blogue, quelqu'un d'autre lisait mes billets avant que je les mette en ligne, ce qui est un bon moyen de savoir si vos explications sont claires. Il arrive que quelque chose qui paraît parfaitement évident et logique à l'auteur soit incompréhensible pour un lecteur qui n'a pas les mêmes acquis ou connaissances sur un sujet. Dit autrement, si un lecteur est incapable de comprendre le sens du billet d'un auteur, c'est d'abord de la faute de l'auteur, c'est-à-dire du manque de clarté dans ce qu'il a écrit.

Si vous avez l'intention d'écrire sur des sujets cliniques et inclure des encadrés concernant des patients, vous devez faire la plus grande attention à préserver l'anonymat des patients; encore mieux: demandez aux patients leur autorisation avant de raconter leur histoire. Il existe de nombreuses anecdotes de médecins qui ont eu le regret de penser avoir correctement déguisé l'identité d'un patient. En conséquence, assurez-vous de lire et respecter toutes les règles éventuelles de votre établissement concernant les médias sociaux.

La capacité d'attention limitée de la majorité des utilisateurs de l'Internet est bien documentée. J'essaie de limiter mes billets à un maximum de 500 mots. Si vous envisagez d'écrire un texte plus long, votre prose a intérêt à être fascinante.

Vous pouvez publier sur votre blogue aussi souvent que vous le souhaitez. En fait, si vous publiez régulièrement, vos lecteurs prendront l'habitude d'aller regarder votre travail. Cela dit, il vaut mieux publier occasionnellement de bons billets que publier fréquemment des billets médiocres.

Il y a deux principaux Sites Web qui soutiennent les blogues: www.blogger.com (Blogger) et www.wordpress. org. Les deux sites Web sont gratuits et ne limitent pas la quantité d'espace utilisable. J'ai commencé avec Blogger et j'y suis resté. L'utilisation de Blogger est un peu déroutante au début, mais comme avec tous les logiciels, plus vous l'utilisez, plus cela vous paraît facile. J'ai donné l'année dernière une conférence sur la façon de commencer un blogue au congrès clinique de l'American College of Surgeons. Des captures d'écran de cette présentation ont été mises en ligne. ${ }^{\mathrm{A}, 2}$

Si vous n'êtes pas sûr(e) de vouloir commencer votre propre blogue, essayez d'envoyer quelques billets en tant qu'invité à des sites tels que KevinMD (http://www. kevinmd.com/blog/) ou The Health Care Blog (http:// thehealthcareblog.com/). Ces deux sites accueillent des contributions.

\section{Et Twitter?}

Oubliez tout ce que vous avez entendu concernant Twitter comme étant une perte de temps. Si vous utilisez Twitter correctement, cela peut faire gagner beaucoup de temps.

Twitter m'aide à rester au courant de ce qu'il se passe dans le monde, notamment en médecine. Vous ne pouvez pas tout lire vous-même. Il y a par exemple plus de 30 journaux et revues sur le seul sujet de l'anesthésiologie. $\mathrm{Si}$ vous suivez les bonnes personnes, elles peuvent vous aider à rester à jour. Vous pouvez utiliser Twitter pour contrôler votre propre flux d'informations.

Quelques spécialités médicales ont trouvé des moyens d'optimiser la valeur de Twitter. Les praticiens de médecine d'urgence ont créé ce qu'ils surnomment «FOAMed » (free open-access medical education: éducation médicale en accès libre). L'utilisation du mot-clé \#FOAMed sur Twitter partagent des liens vers différents articles et blogues. Plusieurs spécialités, dont la chirurgie générale, organisent des clubs Twitter mensuels de journaux auxquels tout le monde peut participer. Un groupe de néphrologues vient d'annoncer la création d'un stage en médias sociaux. ${ }^{2}$ Le site Web digitalsociology.org énumère les multiples raisons pour lesquelles des universitaires (cela s'applique aussi aux médecins) trouvent Twitter utile ${ }^{3}$ comme, par exemple, des réponses rapides à des questions, le fait de rester au courant, le réseautage et ainsi de suite.

Le site Web GCFLearnFree.org propose un didacticiel simple qui montre comment créer un compte Twitter. ${ }^{4}$ Après vous être inscrit sur Twitter, vous pouvez vous faire une idée en «maraudant » ou simplement en observant pendant un moment, et vous n'êtes pas obligés de l'utiliser si vous n'en avez pas envie. Une fois que vous commencez à utiliser Twitter, vos messages sont limités à un maximum de 140 caractères, ce qui vous force à être concis et à apprendre à écrire des titres efficaces.

J'utilise Twitter pour amener du trafic sur mon blogue. Par exemple, pendant un jour ou deux après avoir publié quelque chose de nouveau sur mon blogue, je publie plusieurs fois sur Twitter avec une brève description de ma publication et un lien vers le blogue.

Sree Sreenivasan, ancien directeur numérique de la Columbia University, a expliqué pourquoi Roger Ebert, le critique de films décédé, était un grand utilisateur de Twitter. ${ }^{5}$ Ebert s'intéressait à la vraie vie, communiquait avec les autres et était humble. Il partageait des contenus intéressants et attirait de nombreux suiveurs. Sreenivasan a également observé que les médias sociaux ont changé la façon dont les gens considèrent l'expertise. Par exemple, il ne s'appuie désormais plus seulement sur les revues de films par des critiques bien connus. En revanche, il utilise des sites Web tels que Rotten Tomates (http://www.rottentomatoes.com/) qui compare 
les points de vue des critiques avec ceux des membres du public.

La révision par les pairs après publication est de plus en plus discutée dans les milieux de l'édition médicale et scientifique et va sûrement se développer. Dans le domaine médical, le moteur de recherche PubMed et des journaux tels que le BMJ autorisent des commentaires en ligne sur des articles. Un nouveau site appelé PubPeer (http:// pubpeer.com/) permet de faire des commentaires anonymes sur n'importe quel article. Les blogues et les publications sur Twitter tendent facilement eux-mêmes vers cette nouvelle forme de révision par les pairs après publication.

En résumé, tenir un blogue et utiliser Twitter peut donner de la valeur à votre vie, à la fois sur le plan médical et sur le plan personnel. Veillez simplement à ne pas vous laisser distraire par des vidéos de chats!

Conflicts of interest None declared.

\section{References}

1. Leonard E. WRITERS ON WRITING; Easy on the Adverbs, Exclamation Points and Especially Hooptedoodle. July 16, 2001; Available from URL:http://www.nytimes.com/2001/07/16/arts/writerswriting-easy-adverbs-exclamation-points-especially-hooptedoodle. html (accessed January 2015).

2. Topf $J$. The First Nephrology Social Media Internship. Available from URL: http://www.pbfluids.com/2014/12/the-first-nephrologysocial-media.html (accessed January 2015).

3. Digital Sociology. What Does Twitter Have to Offer Academics? 2013. Available from URL: http://digitalsociology.org.uk/?p=48 (accessed January 2015).

4. GCFLearnFree.org. Twitter 101. Undated. Available from URL: http://www.gcflearnfree.org/twitter101 (accessed January 2015).

5. Sreenivasan S. 5 Social Media Lessons From Roger Ebert, @EbertChicago. 2013; Available from URL: http://www.cnet. com/news/5-social-media-lessons-from-roger-ebert-ebertchicago/ (accessed January 2015).

Conflits d'intérêts Aucun déclaré. 